\title{
Septic arthritis due to Haemophilus influenzae serotype a in the post-vaccination era in Brazil
}

Haemophilus influenzae (Hi) is an important bacterial pathogen in children, causing a variety of respiratory infections and lifethreatening diseases such as meningitis, epiglottitis, pneumonia and septicaemia. Occasionally, Hi can also cause sporadic infections such as septic arthritis (SA). Before the advent of Hi type $b$ (Hib) vaccination, paediatric invasive $\mathrm{Hi}$ disease was caused mostly by Hib isolates. Hib SA in children had been responsible for a significant proportion of cases in Europe and the United States (Bowerman et al., 1997; Peltola et al., 1998; Shoaib et al., 2007).

Finland has the longest experience with Hib conjugate vaccines in Europe, since large-scale vaccination started in 1986. It has been considered that vaccination would probably change the epidemiology and hence the management of SA in children (Peltola et al., 1998) because the importance of non-type $b$ and nonencapsulated (NC) Hi strains could increase (Heath et al., 2001; de Almeida et al., 2005; Goergens et al., 2005).

During the pre-vaccination period (19901999) in Brazil, the annual incidence of meningitis due to Hib in children up to 1 year old and up to 4 years old was 22.3 and 8.8 cases, respectively, per 100000 inhabitants (FUNASA, 2003). In August 1999, the Hib conjugate vaccine was introduced. As of 2002, a new diphtheriatetanus toxoid (DT) whole-cell pertussis combined with Hib conjugated vaccine (DTwP + Hib) was introduced in the National Immunization Program (NIP) in Brazil. Data from the Brazilian National Health Foundation (FUNASA/NIP/ Ministry of Health) show that, in 2002 and 2003, 92.91 and $95.99 \%$, respectively, of eligible children received the vaccine. Since the introduction of the vaccine, only a few papers relating invasive infections with $\mathrm{Hi}$ non-b have been published (Zanella et al., 2002; de Almeida et al., 2005).

This study describes a rare case of septic arthritis due to $\mathrm{Hi}$ serotype a (Hia) in a child vaccinated with a DTwP/Hib conjugate vaccine. The patient was a 3year-old female without previous history of infections admitted to a paediatric hospital of Rio de Janeiro with fever $\left(38{ }^{\circ} \mathrm{C}\right)$, intensive leg pain, restricted hip joint motility and motor difficulties. There was no recent articular trauma. Blood culture for aerobic and anaerobic microorganisms was carried out on admission. Plain radiography revealed no abnormalities. The first blood tests revealed leukocytosis with left shift and sonography imaging detected joint capsule effusion of the right hip. Surgical drainage of the hip was indicated. On the second day, exudate and joint biopsy were sent to the paediatric hospital laboratory for culture. The samples were cultured on brain heart infusion chocolate agar (Difco) enriched with $10 \%$ defibrinated rabbit blood at $37{ }^{\circ} \mathrm{C}$ for $24 \mathrm{~h}$, and bacterial growth was detected after $24 \mathrm{~h}$. Gram staining of bacterial colonies was performed, showing abundant pleomorphic Gram-negative rods, suggesting Haemophilus spp. The suspected culture was send to the National Institute for Quality Control in Health (INCQS) for additional identification and typing tests such as slide agglutination for serotype determination, biotyping, antimicrobial sensitivity and PCR capsular typing as described previously (Falla et al., 1994; Campos, 1999). Blood culture collected on admission was negative after 7 days. Treatment was initiated with $200 \mathrm{mg}$ oxacillin $\mathrm{kg}^{-1} \mathrm{day}^{-1}$, since the initial suspected aetiological agent of the infection was Staphylococcus aureus (Goergens et al., 2005). Antibiotic therapy was changed to oral $30 \mathrm{mg}$ cefuroxime $\mathrm{kg}^{-1}$ day ${ }^{-1}$ after the suspicion of Haemophilus sp. as the causal agent of infection. On the second day, Hia was isolated from the surgical specimens but not from the initial blood culture taken on the day of admission to hospital. After culture confirmation, the antibiotic therapy was changed to parenteral $100 \mathrm{mg}$ of the same drug $\mathrm{kg}^{-1}$ day $^{-1}$ for 20 days. Slide agglutination for serotype, biotyping and PCR capsular typing confirmed Hia biotype III as the causal agent of infection. The Hia strain isolated was a $\beta$-lactamase producer and ampicillin resistant. After antibiotic therapy, the patient showed complete resolution of symptoms, return of normal joint function and normalization of blood parameters.

Since the introduction of Hib conjugate vaccine, the incidence of Hib invasive infections has decreased dramatically. Non-b Hi disease is uncommon in children; however, since the introduction of Hib vaccine, the relative importance of infections due to $\mathrm{NC}$ and non-type $\mathrm{b}$ encapsulated $\mathrm{Hi}$ has increased (Adderson et al., 2001; Heath et al., 2001; Zanella et al., 2002; de Almeida et al., 2005, Ribeiro et al., 2007; Tsang et al., 2007). Adderson et al. (2001) reported an unusual cluster of severe disease caused by Hia with clinical and epidemiological features resembling those of Hib disease. These authors showed that the IS1016-bexA deletion within the capsule gene cassette may identify more virulent strains of Hi. It was suggested that other non-type b strains may then emerge as a cause of invasive infection in the post-vaccination era. In Brazil, rare cases of invasive disease with Hia have been related. In 2002, as part of the Brazilian national epidemiological surveillance laboratories, the Adolfo Lutz Institute (São Paulo) reported 3204 invasive Hi isolates from 1990 to 1999 from blood, cerebrospinal fluid and pleural fluid from different regions of the country. Only 16 of these isolates were Hia. None of these invasive $\mathrm{Hi}$ cases were collected from SA (Zanella et al., 2002). In the city of Salvador, in the north-east of Brazil, active surveillance for meningitis provided evidence of serotype replacement with $\mathrm{Hia}$ after the introduction of the Hib conjugate vaccine, but it was suggested that this was a transient and local phenomenon associated 
with a small increase in meningitis due to two Hia clonal groups (Ribeiro et al., 2007).

It has been suggested that the decrease in Hib carriage due to universal vaccination may have allowed increased colonization with non-type b Hi strains, with the potential to become invasive (WaggonerFountain et al., 1995). The case described here showed that the ability of an Hia strain to emerge as an important pathogen may depend not only on virulence but also on the ability to establish colonization and spread among children vaccinated with $\mathrm{Hib}$ conjugate vaccine. This report also shows the importance of improved diagnostic and characterization procedures in clinical laboratories of public health and of continued Hi surveillance in Brazil. To our knowledge, this is the first report of invasive Hia disease causing SA in Brazil since the introduction of the Hib conjugate vaccine.

\section{Acknowledgements}

This study was supported by FAPERJ/ INCQS/FIOCRUZ/MS. We are grateful to Professor Dr Antonio Campos-Neto (Forsyth Institute, Boston, MA, USA) for a critical review and Raffaella Quental for English revision of the text. All authors report no potential conflicts of interest.

\section{Antonio Eugenio Castro Cardoso de Almeida, ${ }^{1}$ Letícia Ferreira Lima Schroeder, ${ }^{1}$ Nathalia Gonçalves Santos Caldeira, ${ }^{1}$ Nicéa Magaly Matias da Silva, ${ }^{2}$ Paulo Roberto Batista, ${ }^{3}$ Marta Pradel Gallo ${ }^{3}$ and Ivano de Filippis ${ }^{1}$}

${ }^{1}$ Instituto Nacional de Controle da Qualidade em Saúde (INCOS), Departamento de Microbiologia,
Fundação Oswaldo Cruz (FIOCRUZ), Av. Brasil 4365, Manguinhos, 21045-900

Rio de Janeiro, Brazil

${ }^{2}$ Clínica Perinatal de Laranjeiras, Rua das Laranjeiras 455, Laranjeiras, 22240005 Rio de Janeiro, Brazil

${ }^{3}$ Centro Pediátrico da Lagoa, Rua Lineu de Paula Machado 64, J. Botânico, 22470-040 Rio de Janeiro, Brazil

Correspondence: Antonio Eugenio

Castro Cardoso de Almeida

(eugenio.almeida@incqs.fiocruz.br)

Adderson, E. E., Byington, C. L., Spencer, L., Kimball, A., Hindiyeh, M., Carroll, K., Mottice, S., Korgenski, E. K., Christenson, J. C. \& Pavia, A. T. (2001). Invasive serotype a Haemophilus influenzae infections with a virulence genotype resembling Haemophilus influenzae type b: emerging pathogen in the vaccine era? Pediatrics 108, e18.

Bowerman, S. G., Green, N. E. \& Mencio, G. A. (1997). Decline of bone and joint infections attributable Haemophilus influenzae type b. Clin Orthop Relat Res 341, 128-133.

Campos, J. M. (1999). Haemophilus. In Manual of Clinical Microbiology, 7th edn, pp. 604-613.

Washington, DC: American Society for Microbiology.

de Almeida, A. E. C. C., de Filippis, l., de Abreu, A. O., Ferreira, D. G., Gemal, A. L. \& Marzochi, K. B. F. (2005). Occurrence of Haemophilus influenzae strains in three Brazilian states since the introduction of a conjugate Haemophilus influenzae type b vaccine. Braz J Med Biol Res 38, 777-781.

Falla, T. J., Crook, D. W. M., Brophy, L. N., Maskell, D., Kroll, J. S. \& Moxon, E. R. (1994). PCR for capsular typing of Haemophilus influenzae. J Clin Microbiol 32, 2382-2386.

FUNASA (2003). Boletim Eletrônico Epidemiológico. http://www.funasa.gov.br. Accessed 12 June 2003 (in Portuguese).
Goergens, E. D., McEvoy, A., Watson, M. \& Barrett, I. R. (2005). Acute osteomyelitis and septic arthritis in children. J Paediatr Child Health 41, 59-62.

Heath, P. T., Booy, R., Azzopardi, H. J., Slack, M. P. E., Fogarty, J., Moloney, A. C., Ramsay, M. E. \& Moxon, E. R. (2001). Non-type b Haemophilus influenzae disease: clinical and epidemiologic characteristics in the Haemophilus influenzae type b vaccine era. Pediatr Infect Dis J 20, 300-305.

Peltola, H., Kallio, M. J. T. \& Unkila-Kallio, L. (1998). Reduced incidence of septic arthritis in children by Haemophilus influenzae type-b vaccination. J Bone Joint Surg Br 80, 471473.

Ribeiro, G. S., Lima, J. B. T., Reis, J. N., Gouveia, E. L., Cordeiro, S. M., Lobo, T. S., Pinheiro, R. M., Ribeiro, C. T., Neves, A. B. \& other authors (2007). Haemophilus influenzae meningitis 5 years after introduction of the Haemophilus influenzae type b conjugate vaccine in Brazil. Vaccine 25, 4420-4428.

Shoaib, A., Rethnam, U., Bansal, R. \& Clay, N. (2007). The effects of mass immunization of Haemophilus influenzae type B-related orthopaedic disease. J Pediatr Orthop B 16, 236238.

Tsang, R. S. W., Sill, M. L., Skinner, S. J., Law, D. K. S., Zhou, J. \& Wylie, J. (2007).

Characterization of invasive Haemophilus influenzae disease in Manitoba, Canada, 20002006: invasive disease due to non-type b strains. Clin Infect Dis 44, 1611-1614.

Waggoner-Fountain, L. A., Hendley, J. O., Cody, E. J., Perriello, V. A. \& Donowitz, L. G. (1995).

The emergence of Haemophilus influenzae types $\mathrm{e}$ and $\mathrm{f}$ as significant pathogens. Clin Infect Dis 21, 1322-1324.

Zanella, R. C., Casagrande, S. T., Bokermann, S., Almeida, S. C. \& Brandileone, M. C. (2002). Characterization of Haemophilus influenzae isolated from invasive disease in Brazil from 1990 to 1999. Microb Drug Resist 8, 67-72. 